\title{
Numerical modelling of wildland fire spread on the windward and leeward sides of a ridge
}

\author{
C. Simpson $^{\text {a }}$, J. Sharples ${ }^{\mathrm{a}}$ and J. Evans ${ }^{\mathrm{b}}$ \\ ${ }^{a}$ School of Physical, Environmental and Mathematical Sciences, University of New South Wales, Canberra \\ ${ }^{\mathrm{b}}$ Climate Change Research Centre, University of New South Wales, Sydney \\ Email: c.simpson@adfa.edu.au
}

\begin{abstract}
Wildland fires are occasionally observed to spread rapidly across steep leeward slopes, in a direction that is approximately transverse to the background wind. Laboratory experiments and remote sensing of wildland fires suggest that this atypical lateral fire spread can occur regardless of whether the fire is ignited on the leeward slope or advances onto it from the windward slope. The lateral rate of fire spread is typically greatest on the leeward slope close to the ridge, and can considerably affect the subsequent development of the fire, particularly through spotting downwind of the slope. Until now, numerical modelling of this atypical lateral fire spread has considered only the scenario in which a wildland fire is ignited on the leeward slope, with no fire initially present on the windward slope. However, such a scenario will not always occur in reality, and it is fairly common for a wildland fire to cross over from a windward slope to a leeward slope, due to the combined effects of the slope and background wind on the fire spread. It is therefore of interest to establish how a wildland fire can behave as it crosses from a windward to a leeward slope, for a variety of different fire environment conditions, in particular the background wind speed and terrain slope.
\end{abstract}

The aim of this study is therefore to conduct a series of idealised numerical simulations of wildland fire spread, starting from an ignition on a windward slope and with the fire subsequently crossing onto a steep leeward slope. In particular, the analysis focuses on the occurrence of lateral widening of the fire front as it progresses from the windward to the leeward slope, and the relationship of this lateral widening to the background wind speed. As in previous numerical modelling of atypical lateral fire spread, the WRF numerical weather predicition model is used in a large eddy simulation configuration and coupled to the WRF-Fire wildland fire physics module, allowing for direct modelling of the two-way coupled atmosphere-fire interactions. However, in contrast to previous modelling efforts, we amend the way the combined effects of wind and slope on fire spread are modelled in WRF-Fire. This amendment is made to better account for downslope fire spread in particular, though it also offers advantages in other respects.

It has previously been demonstrated that to resolve the fire whirls and turbulent atmospheric eddies that are predominantly responsible for driving the atypical lateral fire spread, is necessary to implement the simulations at high spatial and temporal resolution. In this study we use a spatial resolution of $30 \mathrm{~m}$ and a time step of 0.04 seconds. Simulations were conducted with the fire to atmosphere coupling enabled and disabled to examine the relative effect of coupled fire-atmosphere feedbacks on the lateral fire spread.

The non-coupled simulations failed to produce any significant lateral spread on the windward or leeward slope for any of the background wind speeds considered. In contrast, the coupled simulations exhibited significant lateral spread as the windward slope fire crossed the ridge onto the leeward slope for background wind speeds above $10 \mathrm{~m} \mathrm{~s}^{-1}$. In the coupled simulations the occurrence of lateral spread was strongly associated with the formation of pyrogenic vortices, otherwise known as fire whirls. This is in accordance with previous results concerning the modelling of atypical lateral spread of leeward slope fires.

The modelling results were used to examine the relationship between lateral enhancement of fire spread on the leeward slope and the background wind speed. Raposo et al. (2015) found a power law relationship between the lateral spread enhancement on the leeward slope and the background wind speed in a series of laboratory experiments, as well as in three wildland fire cases. The coupled simulations exhibited a general increase in the lateral enhancement of fire spread for all but the highest background wind speed, though no obvious power law relationship was evident. A number of reasons for the differences in these findings with previous work are briefly discussed.

Keywords: Lateral fire spread, wind-terrain effects, WRF-Fire 


\section{INTRODUCTION}

Countryman (1971) noted that the lee side of a ridge can provide the most favourable situation for the formation of fire whirls, which are rotating columns of air located near or directly above wildland fires. Countryman (1971) noted that although fire whirls often remain stationary, they can also move laterally across leeward slopes or diagonally downslope, contributing to lateral fire spread along the ridge. He cautioned that due to this process, the wind direction should be considered carefully in igniting or fighting lee slope fires. Although knowledge of this process has existed for several decades, until recently there has been little research on the occurrence and effect of lee fire whirls on the behaviour and impact of wildland fires.

A number of wildland fires have recently been observed to have exhibited rapid fire spread in a lateral direction (i.e. approximately transverse to the background wind) on steep leeward slopes (McRae, 2004; Sharples et al., 2012; Raposo et al., 2015). Simpson et al. (2013); Sharples et al. (2013); Simpson et al. (2014) have recently studied this process, sometimes referred to as 'vorticity-driven lateral spread (VLS)', in a series of highly idealised numerical simulations using the Weather Research and Forecasting (WRF) numerical weather prediction model (Skamarock et al., 2008) coupled to a wildland fire physics module (Coen et al., 2013). Under certain fire environment and model conditions, they modelled atypical lateral fire spread on steep leeward slopes that qualitatively matched that described by Countryman (1971).

More recently, Raposo et al. (2015) presented a series of laboratory fires in which the average lateral rate of spread can increase as the fire crosses from a windward slope onto a leeward slope. Additionally, they presented evidence of three wildland fires that had exhibited a similar lateral expansion on the leeward slope, with an apparent dependence of the lateral expansion on the background wind speed in both the laboratory and wildland fires. Their study suggests that atypical lateral fire spread on leeward slopes can occur regardless of whether the fire is initially located on either the windward or leeward slope. However, there are currently no coupled atmosphere-fire numerical simulations against which to compare the results presented by Raposo et al. (2015). It is therefore not clear if the lateral expansion of the fire on the leeward slope is being driven by the same physical process described by Countryman (1971) and Simpson et al. (2013).

The principle aim of this study is therefore to use a coupled atmosphere-fire model, with a similar setup to that used by Simpson et al. (2013); Sharples et al. (2013); Simpson et al. (2014), to examine whether this lateral expansion of a fire as it crosses from a windward to a leeward slope can be reproduced numerically. In addition, there will be a brief examination of the relationship between any lateral fire spread and the background wind speed, to provide a comparison with the results presented by Raposo et al. (2015). In order to complete these numerical simulations satisfactorily, it was necessary to make two minor changes to the fire spread model used in the WRF-Fire wildland fire physics module. These model changes are also described briefly in this paper.

\section{NUMERICAL MODEL DESCRIPTION AND CONFIGURATION}

The numerical simulations presented here were conducted using version 3.6 of the Advanced Research Weather Research and Forecasting (WRF) model (Skamarock et al., 2008). This version of WRF includes the wildland fire physics module, WRF-Fire (Coen et al., 2013), which is a two-dimensional level set method implementation of Rothermel's fire spread model (Rothermel, 1972):

$$
R=R_{0}\left(1+\Phi_{W}+\Phi_{S}\right)
$$

where $R$ is the forward rate of spread and $R_{0}$ is the rate of spread in the absence of wind or slope. The wind and slope correction factors, $\Phi_{W}$ and $\Phi_{S}$, are calculated using the outward pointing normal component of the local slope and mid-flame height winds along each point of the fire line. A logarithmic wind profile is used to determine the mid-flame height winds from WRF. Rothermel's model is semi-empirical and is designed to incorporate radiative and convective heating, contact ignition, short-range spotting and fuel drying.

WRF is used here in a three-dimensional large eddy simulation (LES) configuration (Moeng et al., 2007), allowing it to explicitly resolve the large-scale atmospheric eddies that commonly dominate the planetary boundary layer. Subgrid-scale motions are then modelled using a subfilter-scale stress model. The fire model grid is defined on a 4:1 horizontal refinement of the atmospheric model grid, with a model domain extent of $9 \times 9 \times 5 \mathrm{~km}$ and $300 \times 300 \times 130$ model grid points. The vertical grid spacing is stretched following a hyperbolic tangent function. The simulations are idealised: no radiation physics, microphysics, cumulus parameterisation or planetary boundary layer scheme are used. Diffusion is calculated using the velocity stress tensor, and a prognostic turbulent kinetic energy closure scheme is used to calculate eddy viscosities. Periodic lateral boundaries are used alongside an open radiative upper boundary with a $1 \mathrm{~km}$ deep Rayleigh damping layer. 
Each simulation is initialised using an idealised background wind profile, with a westerly wind, and a potential temperature that is initially set to $300 \mathrm{~K}$ up to a height of $4 \mathrm{~km}$, and increases linearly to $310 \mathrm{~K}$ at the model top of around $5 \mathrm{~km}$. The fire is ignited following a $1 \mathrm{hr}$ period, which allows for the spin-up of background atmospheric turbulence throughout the model domain. The fire is ignited on the windward slope out to a distance of $30 \mathrm{~m}$ in all directions away from a $240 \mathrm{~m}$ long south-north line. The primary model time step is $0.04 \mathrm{~s}$ and a total period of $60 \mathrm{~min}$ of fire spread is modelled, in addition to the spin-up period.

The fire to atmosphere coupling is modelled through the injection of sensible and latent heat into WRF from a parameterised combustion process in WRF-Fire. 17.43 MJ of sensible heat, and a lesser quantity of latent heat, is produced per $1 \mathrm{~kg}$ of fuel combusted in WRF-Fire, with an exponential decrease in fuel mass following a model grid cell's ignition. The sensible and latent heat is calculated at each model time step across the fire model grid, and used to determine the heat flux for each atmospheric model grid column. The sensible and latent heat flux separately affect the potential temperature and water vapour mixing ratio, thereby facilitating modification of the local atmospheric dynamics by the fire. The resulting pyrogenic winds can subsequently affect the modelled fire spread, allowing WRF and WRF-Fire to directly model the two-way coupled atmosphere-fire interactions. The fire to atmosphere coupling can also be disabled, allowing for investigation of fire spread under the background wind and slope. However, as noted by Coen et al. (2013), this will likely result in non-physically realistic fire spread as there is no prescribed fire shape in WRF-Fire.

\section{FIRE SPREAD MODEL}

Viegas (2004) argued that a sufficiently large wildland fire can experience multiple fire spread directions simultaneously due to varying local wind and slope effects. In WRF-Fire, the two-dimensional implementation of Rothermel's fire spread model assumes that for uniform fuels, the fire spread direction and rate of spread at each point along the fire line is determined uniquely by the wind and slope at that model grid cell. In version 3.6 of WRF, two methods are offered for calculating $\Phi_{W}$ and $\Phi_{S}$. First, the local wind and slope are projected onto the outward pointing normal line at each point along the fire line, and those projected values are used to calculate $\Phi_{W}$ and $\Phi_{S}$. Second, $\Phi_{W}$ and $\Phi_{S}$ are calculated using the absolute values for the wind and slope, and then projected onto the outward pointing normal line at the fire line. However, the projected values of $\Phi_{W}$ and $\Phi_{S}$ are individually constrained to be either positive or zero. This prevents $R<R_{0}$, but has some illogical consequences for calculating $R$. For example, under an identical wind speed and direction, a fire will advance downslope at the same rate as along flat terrain, since $\Phi_{S}$ will be zero in both cases. This is inconsistent with empirical data, which indicates that wildland fires typically advance downslope slower compared to over flat
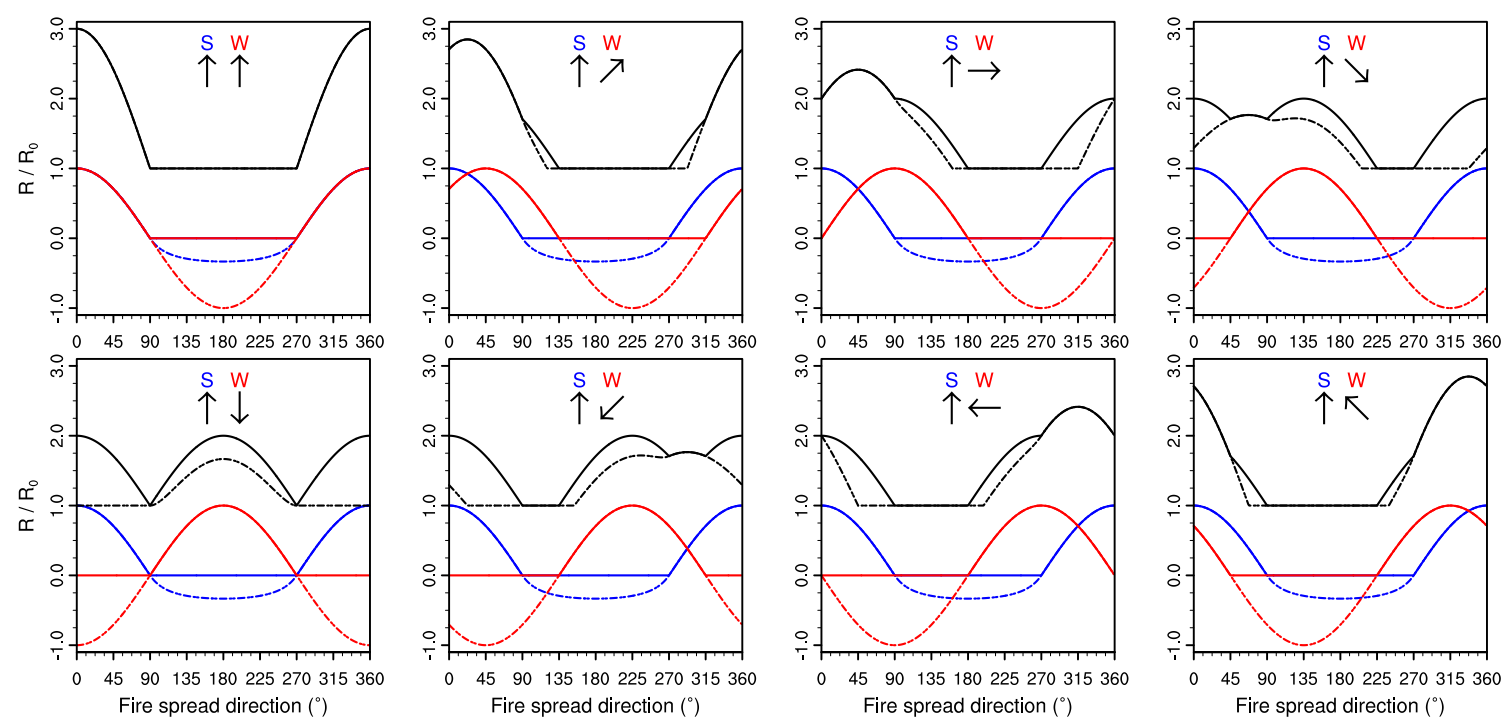

Figure 1. Rate of spread (black) for the original (solid line) and new (dashed line) fire spread models. The slope (blue) and wind (red) contribution factors, are normalised to each have a maximum value of $R_{0}$ when aligned along the fire spread direction, which is measured clockwise from the slope vector, $\mathbf{S}$. The wind vector, $\mathbf{W}$, is shown at $45^{\circ}$ intervals. 
terrain (Weise and Biging, 1997).

In order to address this issue, which will have an important impact in this study as we are considering downslope fire spread on the leeward slope, we propose two modifications to the calculation of $\Phi_{W}$ and $\Phi_{S}$ in WRF-Fire. First, we remove the constraint that they must individually be non-negative and instead impose the constraint that their sum must be non-negative.:

$$
\left(\Phi_{W}+\Phi_{S}\right) \geq 0
$$

Eq 2 ensures that $R \geq R_{0}$, but allows $\Phi_{W}$ and $\Phi_{S}$ to act both constructively and destructively in calculating $R$. Second, we implement the alternative formulation, named the 'kataburn' model, for $\Phi_{S}$ proposed by Sullivan et al. (2014). This formulation reduces $R$ for downslope fire spread and provides a better match with empirical data. Sullivan et al. (2014) initially modified Rothermel's fire spread equation to allow for better comparison with other fire spread models:

$$
\begin{aligned}
R(U, \theta) & =R_{0}\left(\Phi_{W}(U)+\phi_{a}(\theta)\right) & \theta \geq 0 \\
\phi_{a}(\theta) & =1+\Phi_{S}(\theta) &
\end{aligned}
$$

In this study we apply the symmetric hill kataburn-A model, $\phi_{k}^{A}$, on negative slopes $\theta<0$, defined by:

$$
\phi_{k}^{A}(\theta)=\frac{\phi_{a}(-\theta)}{2 \phi_{a}(-\theta)-1} ; \quad \theta<0,
$$

so that the rate of spread on negative slopes is given by

$$
R(U, \theta)=R_{0}\left(\Phi_{W}(U)+\phi_{k}^{A}(\theta)\right) ; \quad \theta<0 .
$$

Substituting Eq 4 into Eq 5 allows for $\phi_{k}^{A}$ to be calculated in terms of $\Phi_{S}$. The combined effect of wind and slope on rate of spread is then given by:

$$
R(U, \theta)=\left\{\begin{array}{l}
R_{0}\left(\Phi_{W}(U)+\frac{1+\Phi_{S}(-\theta)}{1+2 \Phi_{S}(-\theta)}\right) \text { if } \theta<0, \\
R_{0}\left(1+\Phi_{W}(U)+\Phi_{S}(\theta)\right) \text { if } \theta \geq 0,
\end{array}\right.
$$

in combination with the constraint $\mathrm{Eq} 2$.

The effect of these two modifications (Eqs 2 and 7) can be seen in Figure 1, which shows $R, \Phi_{W}$ and $\Phi_{S}$ for both the original and newly proposed methodology. When the wind and slope vectors are aligned, there is no change to $R$ using the new methodology, regardless of the fire spread direction. In all other cases where the wind and slope direction are not identical, $R$ is less than or equal to that calculated using the original methodology. The effect of the kataburn-A model can be seen in $\Phi_{S}$ when the fire spread direction is downslope i.e. between 90 and $270^{\circ}$.

\section{RESULTS AND DISCUSSION}

Following ignition of the fire on the windward slope at the end of the spin-up period, the fire spread is subsequently modelled for $60 \mathrm{~min}$ under one of five different background wind speeds and with the fire to atmosphere coupling either enabled (i.e. coupled) or disabled (i.e. non-coupled). This is sufficient time for the fire to advance up the windward slope and cross the ridge line in each simulation, as shown by Figure 2 .

In each non-coupled simulation, the upslope rate of spread is similar across the width of the fire line, although there is some local variation due to the background atmospheric turbulence. As expected, the upslope rate of spread typically increases with the background wind speed due to an increase in the average $\Phi_{W}$. There is some lateral expansion of the fire on the leeward slope relative to the windward slope, which is accounted for by increased atmospheric turbulence in the lee of the ridge, due to flow separation. To quantify this effect, we calculate the non-dimensional parameter, $k_{3}$, proposed by Raposo et al. (2015). This parameter is calculated using the ratio of the average lateral rate of spread on the leeward and windward side of the ridge. We estimate the lateral rate of spread using the fire spread along the $300 \mathrm{~m}$ terrain height contour on both the windward and leeward slopes. In most cases, this approximately represents the region of greatest lateral fire spread. The parameter $k_{3}$ is estimated to be 1.4 in each non-coupled simulation, as shown in Figure 3, indicating some lateral expansion on the leeward slope, but with no dependence on the background wind speed. 
In contrast, with the fire to atmosphere coupling enabled the fire initially develops a parabolic shape as it advances upslope to the ridge line. In a process qualitatively similar to that described for flat terrain by Clark et al. (1996), there is convergence of the winds ahead of the fire line at the base of the pyro-convective plume. This convergence results in an inwards angling of the winds across the fire line, leading to the parabolic fire shape and a relative increase in the maximum upslope rate of spread compared to the non-coupled simulations (see Figure 2). As was found in the non-coupled simulations, the maximum upslope rate of spread to the ridge line typically increases with the background wind speed, as expected.

In contrast to the unremarkable fire spread on the windward slope, there is a distinct change in the fire spread on the leeward slope, particularly for higher background wind speeds. For a background wind speed of 5 and $7.5 \mathrm{~m} \mathrm{~s}^{-1}$, there is relatively little lateral expansion of the fire on the leeward slope in the coupled simulations compared to the equivalent non-coupled simulations, as demonstrated by Figures 2 and 3. The downslope correction made to the rate of spread by the kataburn model, in addition to the reduced wind speed in the lee of the ridge, result in limited downslope fire spread in both cases. However, as the background wind speed increases to $10 \mathrm{~m} \mathrm{~s}^{-1}$ or higher, the lateral expansion of the fire across the leeward slope begins to increase markedly. This lateral expansion is most evident for the coupled simulation with a background wind speed of $12.5 \mathrm{~m} \mathrm{~s}^{-1}$, with extensive lateral fire spread in both directions and an estimated $k_{3}$ of 2.6. As this lateral expansion at high wind speeds can not be accounted for solely by the background atmospheric conditions, it must result from a modelled dynamic interaction between the fire, the wind and the slope.

Fire whirls are identified in the model output through conditional testing for low local atmospheric pressure, high vertical vorticity and near-surface wind direction reversal around a central point. There is no test for heat flux or temperature, so non-pyrogenic atmospheric vortices can also be occasionally identified. It is apparent in Figure 2 that for high background wind speeds and with the fire to atmosphere coupling enabled, numerous fire whirls are formed in the lee of the ridge. These transient vortices occasionally move across the fire line and contribute considerably towards the lateral expansion of the fire across the leeward slope. In addition, the modelled pyrogenic winds are occasionally aligned with a lateral component across the fire line, and also contribute to the lateral fire spread. This process appears to be at least partly stochastic, as demonstrated by the varying symmetry and asymmetry in the leeward lateral fire spread for the 12.5 and $15 \mathrm{~m} \mathrm{~s}^{-1}$ background wind cases. This process is broadly comparable to that described by Simpson et al. $(2013,2014)$ for fires ignited on the leeward slope, and seems to qualitatively correspond to that described by Countryman (1971).

While the results presented here are broadly consistent with recent numerical modelling studies of leeward

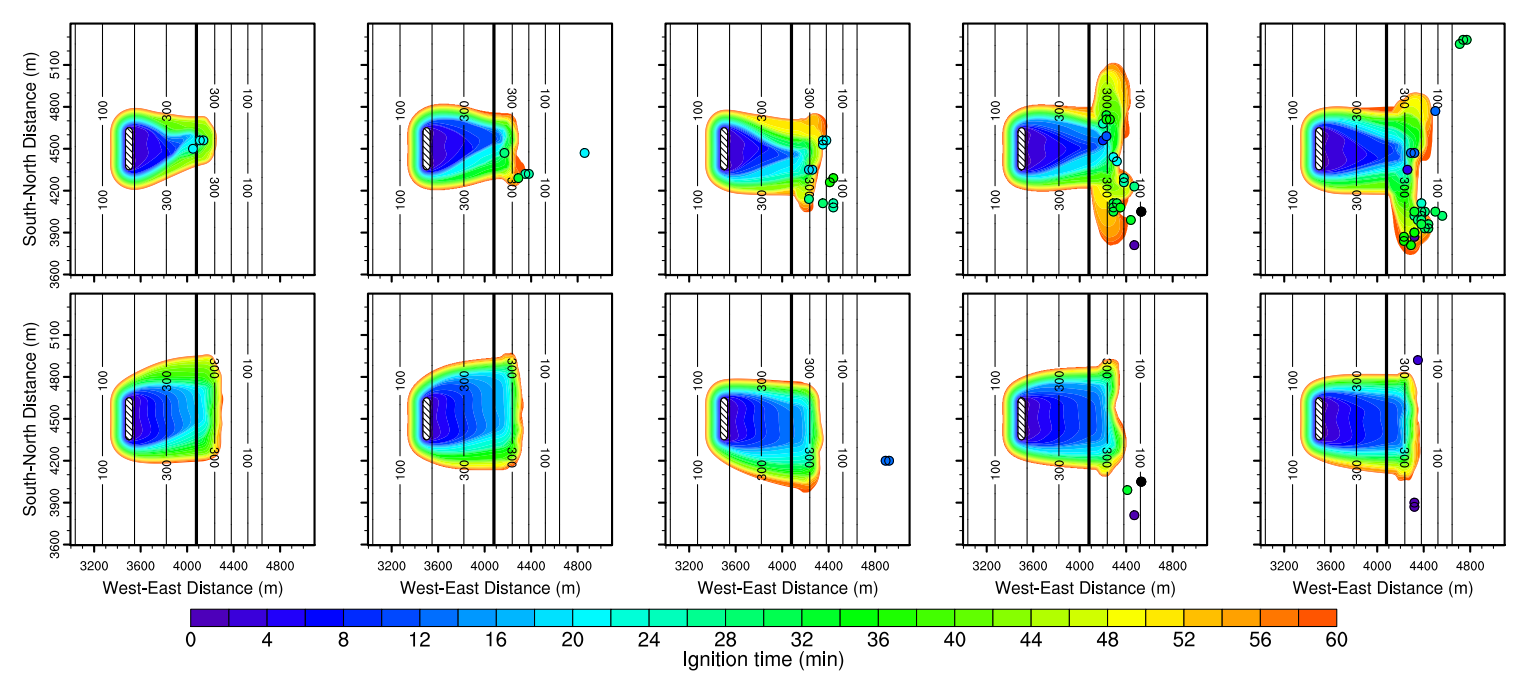

Figure 2. Time of ignition for the coupled (top) and non-coupled (bottom) simulations. The background wind speed increases from left $\left(5 \mathrm{~m} \mathrm{~s}^{-1}\right)$ to right $\left(15 \mathrm{~m} \mathrm{~s}^{-1}\right)$ in increments of $2.5 \mathrm{~m} \mathrm{~s}^{-1}$. The terrain height contours are at $100 \mathrm{~m}$ intervals, and the solid black line shows the location of the ridge line, with a height slightly under $400 \mathrm{~m}$. The circular markers show the time and location of vortices identified by a fire whirl identification algorithm. The dashed region shows the fire ignition region on the windward slope. 


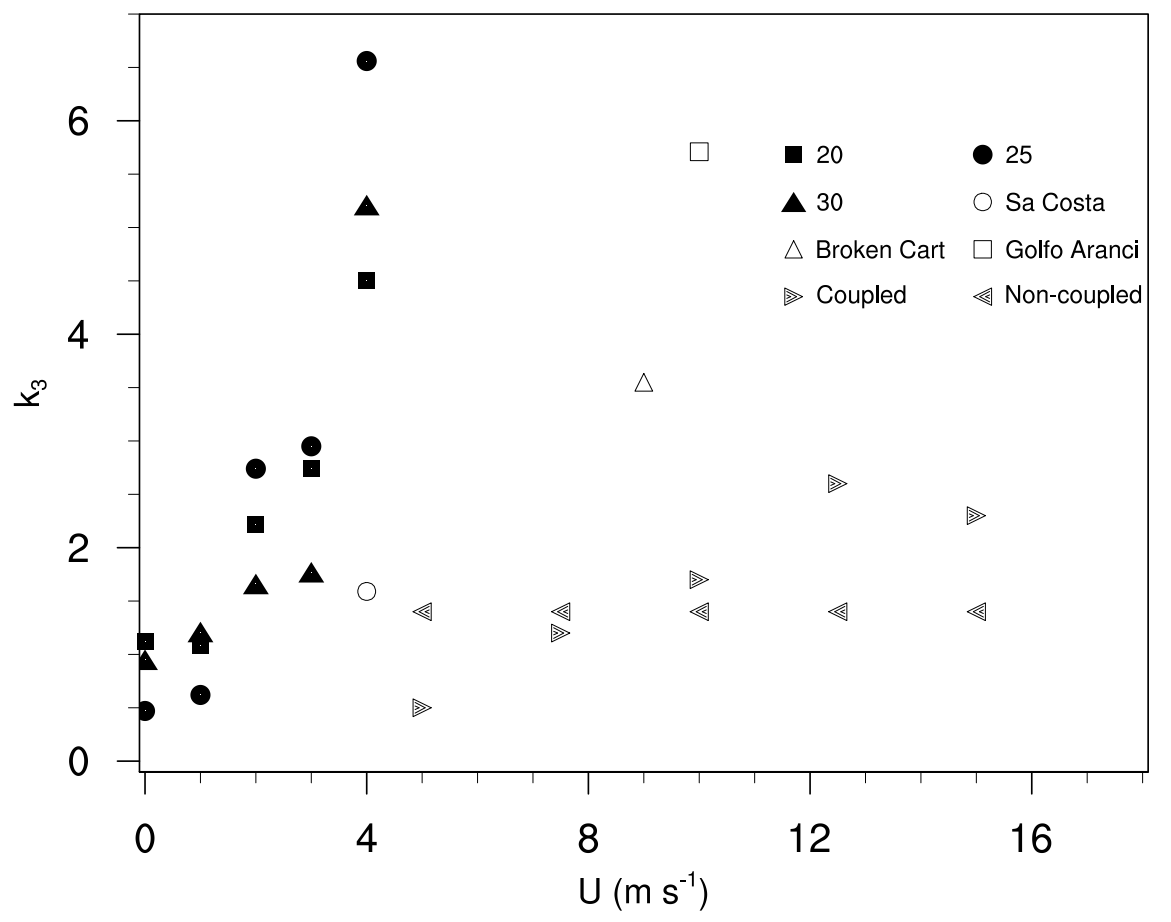

Figure 3. Evolution of the non-dimensional rate of spread parameter, $k_{3}$, with the background wind speed, $U$ $\left(\mathrm{m} \mathrm{s}^{-1}\right)$. The data include laboratory and wildland fires presented by Raposo et al. (2015) (see their Figure $15)$, and the results of the numerical simulations presented in this study.

slope fires, there are some important differences with the windward laboratory and wildland fires discussed by Raposo et al. (2015). In the laboratory fires, the lateral expansion of the fire is typically greatest in the immediate lee of the ridge, whereas in the coupled simulations the point of greatest lateral expansion is typically located around 90 to $120 \mathrm{~m}$ downslope of the ridge, equivalent to around three or four WRF model grid cells. In addition, Raposo et al. (2015) found a power law relationship between the lateral expansion, as quantified by $k_{3}$, and the background wind speed, $U$. It is obvious from Figure 3 that any apparent relationship between $k_{3}$ and $U$ is markedly different for the laboratory fires, the wildland fires, and the five coupled numerical simulations presented here. Raposo et al. (2015) noted that it is difficult to compare the laboratory and wildland fire results directly, as there is considerable uncertainty as to the appropriate method for assigning the background wind velocity in those two cases. It is therefore worth noting that the average mid-flame height wind used to calculate $\Phi_{W}$ in WRF-Fire is considerably lower than the background wind speed aloft assigned to each simulation here. Although there does initially appear to be a positive correlation between $k_{3}$ and $U$ in the coupled simulations, this does not hold true for the $15 \mathrm{~m} \mathrm{~s}^{-1}$ background wind case.

\section{SUMMARY AND CONCLUSIONS}

This study has presented a series of coupled atmosphere-fire numerical simulations of fire spread from a windward slope onto a leeward slope. Similarly to laboratory and wildland fires (Raposo et al., 2015), it was found that the modelled fire can undergo considerable lateral expansion on the leeward slope, with some apparent dependence on the background wind speed. In particular, the numerical simulations suggest that the increased lateral rate of fire spread on the leeward slope can be partly attributed to a combination of fire whirls formed in the lee of the ridge and pyrogenic winds aligned partly transverse to the background wind direction over the fire line.

However, we were not able to reproduce a similar quantitative relationship between the non-dimensional lateral expansion of the fire on the leeward slope and the background wind speed to that proposed by Raposo et al. (2015). There are numerous reasons that could explain the difference in our results: heat release per unit area of combusted fuel (related to fuel type), uniformity of the background wind, ability to resolve lee fire whirls in a physically realistic manner, length of numerical simulation, initial fire shape and proximity to the ridge line, 
terrain variations and roughness length scales. Although it is not possible to explore these possibilities in this study, there is clearly considerable scope for further examination of this relationship in future work.

\section{ACKNOWLEDGEMENT}

This research was supported by the Australian Research Council (ARC) through the Discovery Indigenous Award IN130100038, and was partially supported by the ARC as part of the Future Fellowship FT110100576. The work was also supported by computational resources on the Raijin supercomputer through the National Computational Merit Allocation Scheme. We acknowledge the developers of the NCL and VAPOR data processing and visualization software, which was used in the data analysis.

\section{REFERENCES}

Clark, T. L., M. A. Jenkins, J. Coen, and D. Packham (1996). A coupled atmosphere-fire model: Convective feedback on fire-line dynamics. Journal of Applied Meteorology 35, 875-901.

Coen, J. L., M. Cameron, J. Michalakes, E. G. Patton, P. J. Riggan, and K. M. Yedinak (2013). WRF-Fire: Coupled weather-wildland fire modeling with the Weather Research and Forecasting model. Journal of Applied Meteorology and Climatology 52, 16-38.

Countryman, C. M. (1971). Fire whirls... why, when, and where. Report, USDA Forest Service, Pacific Southwest Forest and Range Experiment Station, Berkeley, CA.

McRae, R. (2004). Breath of the dragon - observations of the january 2003 act bushfires. In Bushfire Conference 2004: Earth, Wind and Fire - Fusing the Elements. South Australian Department of Environment and Heritage.

Moeng, C., J. Dudhia, J. Klemp, and P. Sullivan (2007). Examining two-way grid nesting for large eddy simulation of the PBL using the WRF model. Monthly Weather Review 135(6), 2295-2311.

Raposo, J. R., S. Cabiddu, D. X. Viegas, M. Salis, and J. Sharples (2015). Experimental analysis of fire spread across a two-dimensional ridge under wind conditions. International Journal of Wildland Fire.

Rothermel, R. (1972). A mathematical model for predicting fire spread in wildland fuels. Research Paper INT-115, USDA Forest Service, Intermountain Forest and Range Experiment Station, Ogden, UT.

Sharples, J., R. McRae, and S. Wilkes (2012). Wind-terrain effects on the propagation of large wildfires in rugged terrain: fire channelling. International Journal of Wildland Fire 21, 599-614.

Sharples, J., C. Simpson, and J. Evans (2013). Examination of wind speed thresholds for vorticity-driven lateral fire spread. In J. Piantadosi, R. Anderssen, and J. Boland (Eds.), 20th International Congress of Modelling and Simulation.

Simpson, C. C., J. J. Sharples, and J. P. Evans (2014). Resolving vorticity-driven lateral fire spread using the WRF-Fire coupled atmosphere-fire numerical model. Natural Hazards and Earth System Sciences 14, 2359-2371.

Simpson, C. C., J. J. Sharples, J. P. Evans, and M. F. McCabe (2013). Large eddy simulation of atypical wildland fire spread on leeward slopes. International Journal of Wildland Fire 22, 282-296.

Skamarock, W., J. B. Klemp, J. Dudhia, D. O. Gill, D. M. Barker, M. G. Duda, X.-Y. Huang, W. Wang, and J. G. Powers (2008). A description of the Advanced Research WRF Version 3. Technical Note 475, NCAR.

Sullivan, A. L., J. J. Sharples, S. Matthews, and M. P. Plucinski (2014). A downslope fire spread correction factor based on landscape-scale fire behaviour. Environmental Modelling \& Software 62, 153-163.

Viegas, D. X. (2004). Slope and wind effects on fire propagation. International Journal of Wildland Fire 13, 143-156.

Weise, D. R. and G. S. Biging (1997). A qualitative comparison of fire spread models incorporating wind and slope effects. Forest Science 43(2), 170-180. 